\title{
Compósitos de Borracha Natural com Compostos Condutivos à Base de Negro de Fumo e Polímero Condutor
}

\author{
Marinalva A. dos Santos \\ Departamento de Engenharia de Materiais, UFSCar
}

\author{
Luiz H.C. Mattoso \\ Embrapa Instrumentação Agropecuária
}

\section{Regiani Defácio}

Departamento de Química, Instituto de Pesquisas Tecnológicas

\section{Jamshid Avlyanov \\ Eeonyx Corporation, Pinole, USA}

Resumo: Neste trabalho foram desenvolvidos compósitos condutores elétricos de borracha natural contendo negro de fumo e compostos condutivos baseados em polímeros condutores (Eenomer ${ }^{\circledR}$ ). Os compósitos foram processados a quente num reômetro de torque HAAKE e moldados por prensagem. Foram obtidas placas homogêneas, flexíveis e com ótimo acabamento superficial. Os compósitos foram analisados pelas medidas de torque no processamento, medidas de condutividade elétrica, análise termogravimétrica (TGA), calorimetria diferencial de varredura (DSC) e ensaios de tração. Estes compósitos apresentaram valores de condutividade elétrica entre $10^{-7}$ a $10^{-1} \mathrm{~S} / \mathrm{cm}$, dependendo do tipo de negro de fumo ou composto condutivo utilizado e da quantidade destes no compósito. A análise térmica demonstrou que os compósitos são termicamente estáveis até cerca de $300^{\circ} \mathrm{C}$. Os compostos condutivos atuam como reforço para a borracha natural melhorando suas propriedades mecânicas sem perder significativamente sua flexibilidade.

Palavras-chave: Polímeros condutores, compósitos, negro de fumo, condutividade elétrica, reômetro.

\section{Natural Rubber Composites with Conductive Compounds based on Carbon Black and Conducting Polymers}

\begin{abstract}
In this work, electrically conducting composites of natural rubber with carbon black and natural rubber with conductive compounds containing electrically conducting polymers (Eenomer ${ }^{\circledR}$ ) were developed. The composites were processed in a torque reometer HAAKE and then hot pressed. Homogeneous and flexible plates were obtained with excellent surface finish. The composites were analysed by the torque measurement during processing, electrical conductivity, thermogravimetric analysis (TGA), differential scanning calorimetric (DSC) and mechanical analysis. Conductivity in the order of $10^{-7}$ to $10^{-1} \mathrm{~S} / \mathrm{cm}$ were achieved, depending on the type of carbon black or conductive compound used and their content in the composite. Thermal analysis demonstrated that the compounds are thermally stable until $300^{\circ} \mathrm{C}$. The conductive compounds act as reinforcements in the natural rubber matrix, improving its mechanical properties without significant loss on its flexibility.
\end{abstract}

Keywords: Conducting polymers, composites, carbon black, electrical conductivity, torque rheometry.

Autor para correspôndencia: Luiz H. C. Mattoso, Embrapa Instrumentação Agropecuária, Caixa Postal 741, CEP: 13560-970, São Carlos, SP. E-mail:mattoso@cnpdia.embrapa.br 


\section{Introdução}

A habilidade dos polímeros em atuar como isolantes elétricos é a base de diversas aplicações elétricas e eletrônicas. No entanto, são muitos também os casos em que a condutividade elétrica dos materiais poliméricos é requerida, tais como para dissipação de carga estática, proteção contra interferência eletromagnética e embalagens protetoras para componentes eletrônicos ${ }^{[1-3]}$. Nestes casos, a Engenharia de Materiais tem procurado combinar a versatilidade dos polímeros com as propriedades elétricas dos metais. As vantagens dizem respeito não só à capacidade para produzir materiais condutores elétricos, mas também à possibilidade para modificar suas propriedades em ampla faixa.

O método geralmente usado para aumentar a condutividade elétrica dos polímeros é através da incorporação de aditivos condutivos específicos a estes, como pós metálicos, fibras metálicas, polímeros condutores iônicos e polímeros intrinsecamente condutores. Nos compósitos de borracha com negro de fumo, as partículas de negro de fumo tendem a formar na matriz isolante, trilhas condutoras, que dependem da quantidade da carga utilizada. Em baixas concentrações de negro de fumo, a resistividade das composições é essencialmente a do meio isolante. Com o aumento do teor de carga, um valor de concentração crítica é atingida, a partir do qual a resistividade começa a decrescer rapidamente, chamado composição de percolação, devido à formação de um caminho condutor dentro da matriz isolante ${ }^{[4-6]}$.

Os polímeros intrinsecamente condutores possuem grande potencial para aplicações tecnológicas. No entanto, atualmente o processamento em larga escala pelos métodos usados na indústria de artefatos poliméricos ainda está sendo desenvolvido ${ }^{[5,7]}$. A preparação de blendas e compósitos de polímeros condutores com polímeros convencionais é um método bastante promissor, pois permite a combinação da condutividade elétrica dos polímeros condutores com as propriedades mecânicas e térmicas dos polímeros convencionais, aumentando o potencial tecnológico destes materiais. Entretanto, embora estas blendas apresentem vantagens de melhor desempenho mecânico sobre os polímeros condutores sozinhos ${ }^{[2,5,7-8]}$, existem poucos estudos sistemáticos para o processamento destes materiais por fusão, uma vez que a fabricação de blendas por solução, em geral, não é um processo industrialmente viável. A maior dificuldade envolven- do a fabricação de blendas condutoras elétricas usando polianilina e polipirrol é a instabilidade térmica destes polímeros no estado dopado na temperatura de processamento ${ }^{[8-10]}$. Além disso, a polianilina condutiva é ácida, em geral, e os ajustes dessa acidez para valores de $\mathrm{pH}$ neutro elimina sua condutividade ${ }^{[11,12]}$.

Os compostos Eeonomer ${ }^{\circledR}$ são uma nova classe de aditivos condutivos, obtidos pela polimerização in-situ de polímeros intrinsecamente condutores (polianilina ou polipirrol) sobre o negro de fumo. Estes compostos, produzidos em pH neutro, são termicamente estáveis e compatíveis com as condições de processamento por fusão de polímeros. Estudos da formulação destes com termoplásticos indicam melhores propriedades elétricas, mecânicas e de processamento, quando comparadas com as propriedades obtidas da formulação com aditivos condutores tradicionais. A alta estabilidade térmica e a condutividade destes compostos contendo polímero condutor são independentes do $\mathrm{pH}$, e a sua condutividade elétrica permanece relativamente inalterada numa faixa de $\mathrm{pH}$ de 0 a 8 .

Este trabalho tem como objetivo desenvolver, caracterizar e comparar as propriedades de compósitos condutores elétricos de borracha natural com negro de fumo, e borracha natural com um novo tipo de composto condutivo contendo polímeros condutores, Eeonomer $^{\circledR}$. As propriedades elétricas, térmicas e mecânicas destes compósitos serão analisadas e correlacionadas.

\section{Experimental}

\section{Materiais}

A borracha natural (NR) utilizada foi do tipo Crepe Clara Brasileira (CCB-1), fornecida pela Borracha Paulista Indústria e Comércio.

Foram utilizados dois tipos de negro de fumo: a) condutivo XE2/PRINTEX XE2 da Degussa (tamanho de partícula de $35 \mathrm{~nm}$, área superficial específica de $1000 \mathrm{~m}^{2} / \mathrm{g}$ e densidade de $0,10 \mathrm{~g} / \mathrm{cm}^{3}$ ) e b) condutivo KP0/Ketjenblack EC600jd da AKZO NOBEL (tamanho de partícula de $40 \mathrm{~nm}$, área superficial específica de $1400 \mathrm{~m}^{2} / \mathrm{g}$ e densidade de $0,11 \mathrm{~g} / \mathrm{cm}^{3}$ ).

O compostos condutivos contendo polímeros condutores utilizados foram:

Eeonomer ${ }^{\circledR} \mathrm{P} 20-5 \mathrm{DB} / \mathrm{S}$ (tamanho de partícula de 40nm, área superficial específica de $320 \mathrm{~m}^{2} / \mathrm{g}$ ).

Eeonomer ${ }^{\circledR}$ KPY-20-7D (tamanho de partícula de 
40nm, área superficial específica de $390 \mathrm{~m}^{2} / \mathrm{g}$ e densidade de $\left.0,062 \mathrm{~g} / \mathrm{cm}^{3}\right)$.

Eeonomer ${ }^{\circledR}$ KP20-7DNF (tamanho de partícula de $40 \mathrm{~nm}$, área superficial específica de $570 \mathrm{~m}^{2} / \mathrm{g}$ e densidade de $0,062 \mathrm{~g} / \mathrm{cm}^{3}$ ).

Os compostos condutivos descritos acima são de uma nova geração de compostos de negro de fumo modificados pela deposição superficial de polímero intrinsecamente condutor. Eeonomer ${ }^{\circledR} \mathrm{P} 20-5 \mathrm{DB} / \mathrm{S}$ é um composto formado pelo negro de fumo XE2 revestido com polianilina dopada com dopante sulfônico. O composto Eeonomer ${ }^{\circledR}$ KPY-20-7D é formado do negro de fumo KP0 com $20 \%$ de polipirrol dopado com dopante sulfônico. O composto Eeonomer ${ }^{\circledR} \mathrm{KP} 20-7 \mathrm{DNF}$ é formado do negro de fumo KP0 com $20 \%$ de polianilina dopada com dopante sulfônico. Todos os compostos condutivos e os negros de fumo utilizados foram fornecidos pela Eeonyx Co. (USA).

Foi utilizado como antioxidante o 1,4 diamino benzeno, fornecido pela indústria Flexsys. O acelerador (dissulfeto de 2,2'-dibenzotiazol - MBTS) e os demais aditivos (óxido de zinco, ácido esteárico e enxofre) foram fornecidos pela indústria Petroflex Ind. e Com S/A. Todos os aditivos foram utilizados conforme recebidos.

\section{Preparação dos Compósitos}

A formulação básica dos compósitos de borracha natural-negro de fumo e borracha natural-composto condutivo está indicada na Tabela 1 .

O processamento dos compósitos condutivos foi realizado em uma câmara de mistura interna de um

Tabela 1. Formulação para compósitos de borracha natural-negro de fumo e borracha natural-composto condutivo.

\begin{tabular}{lc}
\hline \multicolumn{1}{c}{ Componentes } & Quantidade (phr) \\
\hline Borracha natural (NR) & 100 \\
Ácido esteárico & 5 \\
Óxido de zinco & 5 \\
Óleo de processamento & variável (5 a 30) \\
Composto condutivo & variável (5 a 30) \\
Negro de fumo condutivo & 2 \\
Dissulfeto de 2,2' - dibenzotiazol & 1 \\
1,4 diamino benzeno & 2,5 \\
Enxofre
\end{tabular}

Reômetro de Torque HAAKE. Foram testadas algumas condições de processamento dos compósitos de borracha natural com negro de fumo ou composto condutivo, e selecionadas as seguintes condições de processamento: temperatura de $70^{\circ} \mathrm{C}$, velocidade de rotação de $80 \mathrm{rpm}$ e tempo de mistura de 15 minutos. Após o processamento no HAAKE o acelerador e o enxofre foram incorporados à mistura, através de um moinho de dois rolos, a uma temperatura de $\approx 50^{\circ} \mathrm{C}$. Após $24 \mathrm{~h}$ foi feita a moldagem por prensagem a quente a uma temperatura de $\approx 145^{\circ} \mathrm{C}$ e uma pressão de $\approx 4$ MPa durante 20 min em uma prensa marca Luxor, modelo LPB-35-15. A temperatura e o tempo de prensagem foram definidos como a condição onde ocorre a vulcanização completa dos compósitos, segundo dados de análise de cura obtidos em um reômetro de torque Monsanto.

\section{Caracterização dos Compósitos}

As medidas de condutividade elétrica nas amostras condutivas foram realizadas através do método de quatro pontas e nas amostra de NR pura foi utilizado o método de duas pontas. Para as medidas de Calorimetria Diferencial de Varredura (DSC) foi utilizado um instrumento marca Du Pont modelo 2000 com uma taxa de aquecimento de $10^{\circ} \mathrm{C} / \mathrm{min}$ em uma atmosfera dinâmica de nitrogênio, em uma faixa de temperatura de $-100^{\circ} \mathrm{C}$ a $300^{\circ} \mathrm{C}$. As análises termogravimétricas foram realizadas em termobalança TG 209 (NETZSCH), com início à temperatura ambiente até $600^{\circ} \mathrm{C}$, a uma taxa de aquecimento de $10^{\circ} \mathrm{C} / \mathrm{min}$, em atmosfera dinâmica de nitrogênio.

Os Ensaios de Tração foram realizados segundo a ASTM D 412 - 97, Método A, em um equipamento marca EMIC, em pelo menos cinco amostras para cada tipo de compósito. Foram considerados os resultados em que os filmes não romperam próximos às garras. A velocidade de ensaio utilizada foi de 500 $\mathrm{mm} / \mathrm{min}$ à temperatura ambiente.

\section{Resultados e Discussão}

Torque

Com o objetivo de analisar os compósitos de borracha natural-negro de fumo e borracha natural-composto condutivo contendo polímero condutor foi avaliada a variação do torque durante o processamento no reômetro para cada compósito de borracha natu- 
Tabela 2. Valores de torque total e temperatura no final do processamento no reômetro de torque dos compósitos de borracha natural e compostos condutivos.

\begin{tabular}{cccc}
\hline $\begin{array}{c}\text { Tipo de } \\
\text { composto } \\
\text { condutivo }\end{array}$ & $\begin{array}{c}\text { Conteúdo } \\
\text { de composto } \\
\text { condutivo na } \\
\text { borracha } \\
\text { (phr) }\end{array}$ & $\begin{array}{c}\text { Torque total } \\
(\mathbf{N m})\end{array}$ & $\begin{array}{c}\text { Temperatura } \\
\text { final } \\
\left({ }^{\circ} \mathbf{C}\right)\end{array}$ \\
\hline nenhum & 0 & 1050 & 92 \\
\hline XE2 & 5 & 1650 & 92 \\
\hline KPO & 5 & 1750 & 106 \\
\hline KP20 & 20 & 1700 & 94 \\
& 5 & 1650 & 93 \\
\hline KPY & 5 & 1800 & 107 \\
\hline & 20 & 1350 & 90 \\
\hline
\end{tabular}

ral. Pode-se observar na Tabela 2 que, para um mesmo compósito, variando a quantidade de negro de fumo ou composto condutivo, o torque e a temperatura final aumentam com o aumento na quantidade destes no compósito.

Comparando os diferentes tipos de compósitos, para uma mesma composição, observa-se que o compósito com negro de fumo (NR/KP0-5phr) apresenta o maior valor de torque $(\approx 1700$ N.m) e o maior valor de temperatura $\left(\approx 94^{\circ} \mathrm{C}\right)$, ao final do processamento, enquanto que o compósito contendo polipirrol (NR/KPY-5phr) apresenta o menor valor de torque $(\approx 1350$ N.m $)$ e o menor valor de temperatura $\left(\approx 90^{\circ} \mathrm{C}\right)$ ao final do processamento. Os maiores valores de torque e temperatura, nos compósitos NR/KP0, ocorrem pelo fato do negro de fumo destes compósitos apresentarem área superficial (KPO- $1400 \mathrm{~m}^{2} / \mathrm{g}$ ) maior que a dos compostos contendo polímero condutor (KPY$390 \mathrm{~m}^{2} / \mathrm{g}, \mathrm{KP} 20-570 \mathrm{~m}^{2} / \mathrm{g}$ e P $20-320 \mathrm{~m}^{2} / \mathrm{g}$ ). O aumento da área superficial causa um aumento de cisalhamento que gera dissipação de energia na forma de calor. Desse modo, tem-se o aumento do torque e da temperatura. Segundo Avlyanov et alli ${ }^{[13-15]}$ o processamento por fusão de negro de fumo altamente estruturado é difícil nos compostos termoplásticos, devido ao aumento da viscosidade, que está relacionado à alta área superficial do negro de fumo. A deposição de polipirrol e polianilina sobre o negro de fumo resulta numa redução efetiva da área superfi- cial. A redução da área superficial facilita o processamento dos compostos contendo polímero condutor, melhorando também os parâmetros de fluxo do fundido dos compósitos condutivos quando comparados aos compósitos carregados só com negro de fumo. Este resultado representa uma vantagem muito importante, do ponto de vista reológico, dos compostos condutivos contendo polímero condutor, pois os menores valores de torque podem levar a uma economia de energia e maior facilidade na etapa de processamento.

Para um mesmo tipo de composto condutivo observa-se que quando se aumenta a quantidade deste na composição ocorre também um aumento do torque, devido ao aumento do número de "sítios" de ancoramento da borracha natural e consequentemente do reforço da mesma. Verifica-se também que os compósitos com negro de fumo (NR/KP0) reforçam mais a borracha natural do que os compósitos com composto condutivo (NR/KPY e NR/KP20), pelo fato de estes últimos apresentarem uma quantidade razoável de polímero intrinsecamente condutor (polipirrol e polianilina), de aproximadamente $20 \%$, que se localiza na superfície do negro de fumo, diminuindo sua porosidade superficial e consequentemente reduzindo também o número de 'sítios' de ancoramento das macromoléculas da matriz borrachosa.

\section{Caracterização Elétrica}

A Figura 1 mostra as curvas de condutividade elétrica versus quantidade de negro de fumo ou composto condutivo contendo polímero condutor, para os compósitos: NR/XE2, NR/KP0, NR/KP20, NR/P20 e NR/KPY. A condutividade elétrica da borracha natural, determinada pelo método de duas pontas, é da ordem de $10^{-12} \mathrm{~S} / \mathrm{cm}$. Através da análise das curvas, observa-se que a incorporação de compostos condutivos promove um aumento na condutividade elétrica para até $\approx 1 \mathrm{~S} / \mathrm{cm}$, nas condições estudadas. O compósito NR/KPO apresenta o menor limite de percolação $\left(\approx 10^{-2} \mathrm{~S} / \mathrm{cm}\right.$, para $10 \mathrm{phr}$ de KPO), e o composto NR/P20 apresenta o maior limite de percolação (da ordem de $10^{-3} \mathrm{~S} / \mathrm{cm}$, para $25 \mathrm{phr}$ de KP20). Segundo Wessling ${ }^{[7]}$ o comportamento de percolação do negro de fumo apresenta uma dependência da área superficial. Negros de fumo com maior área superficial apresentam um menor limite de percolação, possuindo uma maior superfície líquida acessível à matriz e assim serão melhor dispersados. Nos compósitos 


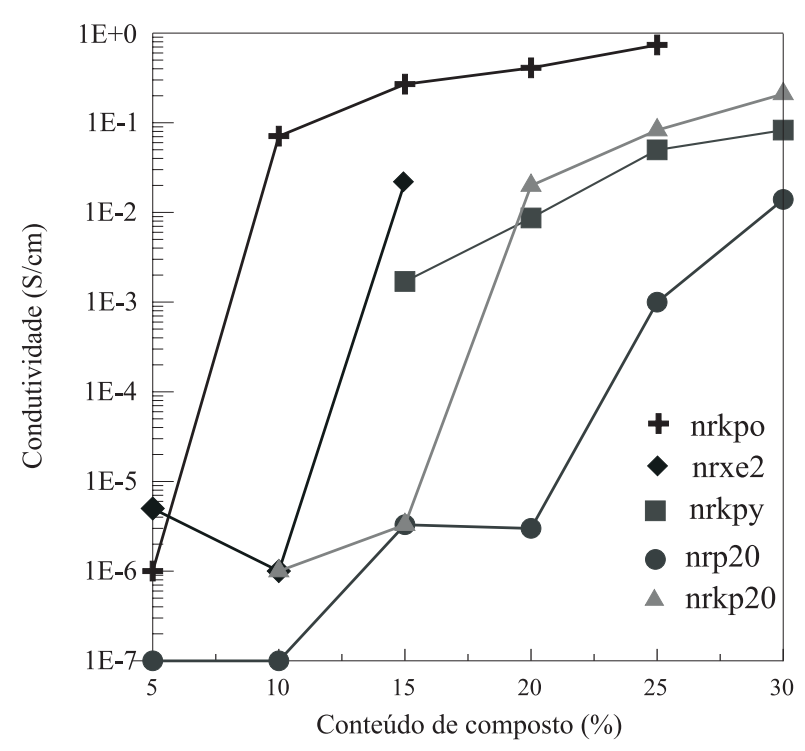

Figura 1 - Curvas de condutividade elétrica versus conteúdo de composto condutivo, para os diferentes compósitos com borracha natural: NR/XE2, NR/KPO, NR/KP20, NR/P20 e NR/KPY, conforme indicado.

estudados observa-se que o compósito NR/KPO é o que apresenta os maiores valores de condutividade elétrica, seguido pelos compósitos NR/KP20 e NR/KPY. Através dos dados de área superficial apresentados na seção experimental observa-se que o negro de fumo KPO possui a maior área superficial dentre os compostos estudados. No entanto deve-se lembrar que, embora os compósitos com negro de fumo sejam mais condutivos, o seu processamento é mais difícil em relação aos compostos contendo polímero condutor.

Estes resultados de processamento e condutividade elétrica são bastante importantes, principalmente se comparados com os dos compósitos condutivos de negro de fumo com algumas borrachas $^{[16-21]}$ e de algumas blendas condutivas de polianilina e polipirrol ${ }^{[22-25]}$. No primeiro caso necessita-se de uma grande quantidade de negro de fumo para se obter uma condutividade elétrica razoável, causando-se prejuízos nas propriedades mecânicas e dificuldades de processamento. No segundo caso a literatura tem demonstrado que há grande dificuldade no processamento de blendas com polímeros intrinsecamente condutores (polianilina e polipirrol) pelos métodos usuais de processamento, tendo sido obtidos, em geral, valores bastante inferiores aos do presente trabalho. Os valores de condutividade elétrica obtidos neste trabalho estão na faixa dos semicondutores ${ }^{[7]}$ com uma larga faixa de aplicação para esses compósitos, como dissipação de carga es- tática e proteção contra interferência eletromagnéti$\mathrm{ca}^{[1-3,26-28]}$.

\section{Análise Térmica}

A Figura 2 apresenta os resultados das análises de TGA para a borracha natural (NR), para os compósitos NR/KPO, NR/XE2, NR/KPY, e NR/KP20 com 20phr de composto condutivo. Nenhuma perda de massa significativa foi observada na faixa da temperatura ambiente até cerca de $200^{\circ} \mathrm{C}$, pois os compostos condutivos foram secos antes do processamento $\left(125^{\circ} \mathrm{C}, 1 \mathrm{~h}\right)$. As curvas de TGA dos compostos condutivos antes da secagem demonstraram uma perda de massa de cerca de $8 \%$ e nenhuma perda de dopante até $300^{\circ} \mathrm{C}$. Analisando a quantidade de perda de massa na faixa de temperatura de 200 a $300^{\circ} \mathrm{C}$, para os compósitos estudados, observa-se que: os compósitos NR/KPO-20phr e NR/XE2-20phr apresentaram uma perda de massa de aproximadamente 5\%, e os compósitos NR/KPY-20phr e NR/KP20$20 \mathrm{phr}$ apresentaram uma perda de massa de cerca de $4 \%$. Nos resultados obtidos por Wei et alli ${ }^{[29]}$, estudando filmes de polianilina dopados com $\mathrm{HCl}$, houve perda significativa de massa à temperatura em torno de $200^{\circ} \mathrm{C}$, atribuída à perda do dopante $(\mathrm{HCl})$. Estudos em blendas de polianilinas ${ }^{[5,8]}$ demonstram que, em geral, a estabilidade térmica é aumentada para cerca de $250^{\circ} \mathrm{C}$ pela dopagem com ácidos sulfônicos e a perda de massa diminuída pela adição de um polímero convencional estável. Analisando os resultados da temperatura de início de degradação dos compósitos verifica-se que os compósitos NR/KPO20phr e NR/XE2-20phr apresentam uma temperatu-

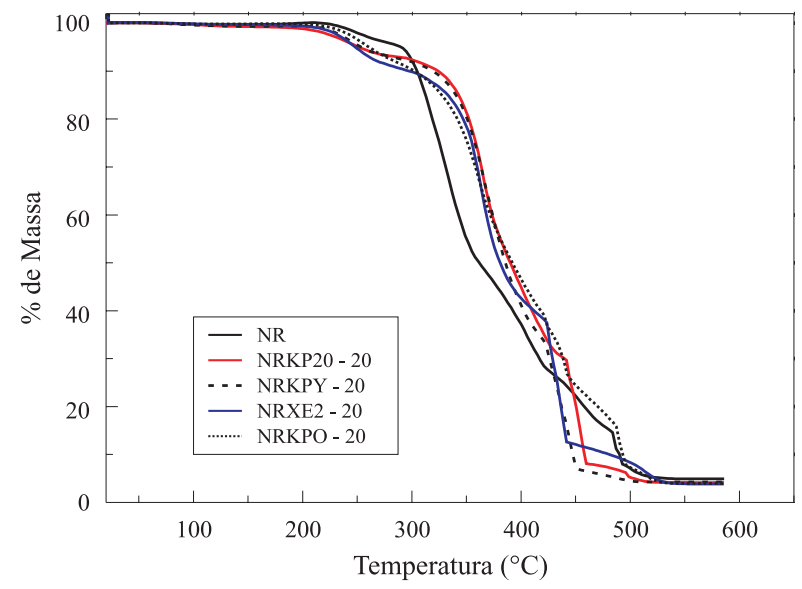

Figura 2. Curvas de TGA para borracha natural (NR) e para os compósitos NR/KPO-20phr, NR/XE2-20phr, NR/KP2O-20phr e NR/ KPY-20phr. 
ra de início de degradação de $\approx 300^{\circ} \mathrm{C}$, e os compósitos NR/KPY-20phr e NR/KP20-20phr apresentam uma temperatura de início de degradação $\approx 330^{\circ} \mathrm{C}$. Comparando os resultados de perda de massa obtidos verifica-se que os compósitos com negro de fumo (NR/KPO-20phr e NR/XE2-20phr) apresentam maior perda de massa em relação aos compósitos com polímero condutor (NR/KP20-20phr e NR/KPY-20phr).

Estes resultados são importantes, pois comprovam a hipótese de Avlyanov et alli[i ${ }^{[14]}$ de que os compostos condutivos contendo polímero condutor são termicamente estáveis nas temperaturas de processamento de polímeros. No nosso estudo esses compostos proporcionam maior estabilidade térmica a borracha natural do que os negros de fumo sem polímero condutor. Isto sugere que os ancoramentos entre as macromoléculas da borracha natural e as partículas dos compostos condutivos contendo polímero condutor, originados durante o processamento a quente no HAAKE, são mantidas mesmo após as altas temperaturas de processamento. Segundo a literatura ${ }^{[14,30]}$ as razões para o aumento da estabilidade térmica dos compostos contendo polímero condutor pode ser explicado pela interação múltipla $\pi$ - $\pi$ entre as ligações insaturadas dos anéis dos polímeros condutores e os anéis aromáticos do negro de fumo e os anéis aromáticos planares dos íons dopantes. Estas interações limitam a livre rotação dos segmentos do polímero condutor conjugado e do dopante, aumentando a estabilidade térmica destes.

Na Figura 3 são mostrados os resultados das análises de DSC para os compósitos NR/KPO, NR/XE2, NR/KPY e NR/KP20 com 20phr de composto condutivo na faixa de temperatura de -100 a $20^{\circ} \mathrm{C}$. Verifica-se que todas as curvas apresentam um pico

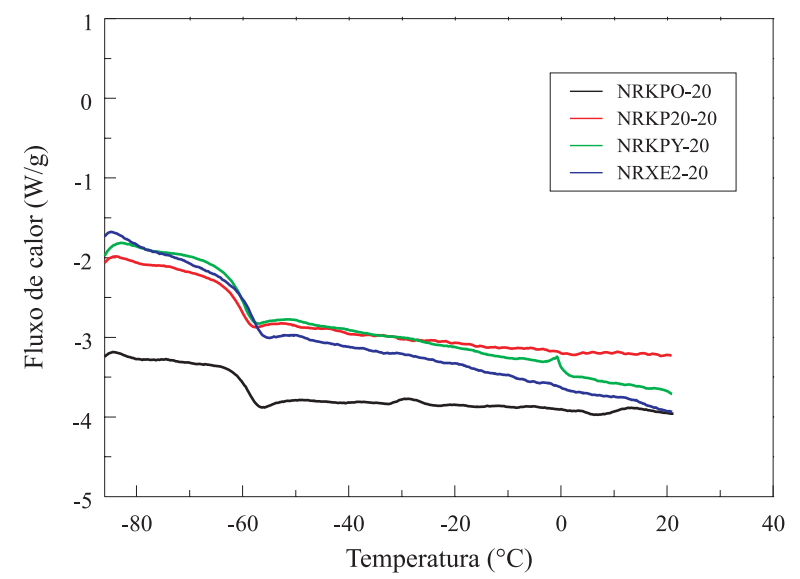

Figura 3 - Curvas de DSC de diferentes compósitos de borracha natural NR/KPO-20phr, NR/KPY-20phr, NR/XE2-20phr e NR/KP20-20phr. de transição na faixa de temperatura entre $-68^{\circ} \mathrm{C}$ e $-55^{\circ} \mathrm{C}$, que atribuímos à temperatura da transição vítrea $(\mathrm{Tg})$ da borracha natural. Observa-se também que as curvas referentes aos compósitos NR/KPY-20phr e NR/KP20-20phr apresentam valores de Tg ligeiramente menores $\left(\approx-62^{\circ} \mathrm{C}\right)$ do que a dos compósitos com negro de fumo $\left(\approx-58^{\circ} \mathrm{C}\right)$. Estes resultados são consistentes com o menor efeito reforçante dos compostos condutivos com polímero condutor.

Estes compostos foram também analisados por DSC na faixa de temperatura de $20^{\circ} \mathrm{C}$ a $300^{\circ} \mathrm{C}$, não demonstrando nenhuma transição aparente nesta faixa, confirmando que os compósitos com polímero condutor são estáveis termicamente até $300^{\circ} \mathrm{C}$ de acordo com os resultados obtidos na análise feita por TGA.

\section{Ensaios de Tração}

Para avaliar as propriedades mecânicas dos compósitos obtidos, foram feitos ensaios de tração na formulação borracha natural sem carga e nos compósitos NR/KPY, NR/KP20, NR/P20, NR/KP0 e NR/XE2. A Tabela 3 mostra os valores médios e desvio padrão da tensão na ruptura $(\sigma)$ e da deformação na ruptura $(\varepsilon \%)$ para os compósitos estudados, nas composições de 5, 10, 15, 20, 25 e 30phr. Comparando-se os resultados da Tabela 3 , verifica-se que os compósitos de borracha natural (NR) com o negro de fumo KP0 (NR/KP0) apresentam, em geral, maiores valores de tensão na ruptura do que os compósitos NR/XE2, NR/KP20, NR/P20 e NR/KPY. Para os compósitos de borracha natural (NR) com compostos condutivos contendo polímero condutor, observa-se que os compósitos contendo polianilina (NR/KP20 e NR/P20) apresentam valores de tensão na ruptura maiores que os valores dos compósitos contendo polipirrol (NR/KPY).

Fazendo-se uma análise da tensão na ruptura e da deformação na ruptura dos valores obtidos para os compósitos estudados e os valores da borracha natural pura (sem carga condutiva), verifica-se que o compósito $\mathrm{NR} / \mathrm{KP} 0$ apresenta maiores valores de tração do que a NR pura, e menor deformação percentual. Já os compósitos com composto condutivo contendo polímero condutor NR/KP20 e NR/KPY apresentaram menores valores tanto da tensão na ruptura como da deformação percentual, devido ao seu menor caráter reforçante provocado pela diminuição da área superficial. Estes resultados estão de acordo com o verificado na literatu$\mathrm{ra}^{[31]}$, no estudo das propriedades mecânicas de blendas 
Santos, M. A. et al. - Compósitos de borracha natural com compostos condutivos

Tabela 3. Resultados dos ensaios de tração (valores médios e desvio padrão da tensão na ruptura $(\sigma)$ e da deformação na ruptura $(\varepsilon)$ ) dos compósitos NR/KPY, /KP20, NR/P20, NR/KP0 e NR/XE2.

\begin{tabular}{|c|c|c|c|c|c|c|c|c|c|c|}
\hline \multirow{2}{*}{$\begin{array}{c}\text { Conteúdo de } \\
\text { composto condutivo } \\
\text { (phr) }\end{array}$} & \multicolumn{2}{|c|}{ NR/KPY } & \multicolumn{2}{|c|}{ NR/KP20 } & \multicolumn{2}{|c|}{ NR/P20 } & \multicolumn{2}{|c|}{ NR/KP0 } & \multicolumn{2}{|c|}{ NR/XE2 } \\
\hline & $\begin{array}{c}\sigma \\
(\mathrm{MPa})\end{array}$ & $\begin{array}{c}\varepsilon \\
(\%)\end{array}$ & $\begin{array}{c}\sigma \\
(\mathrm{MPa})\end{array}$ & $\begin{array}{c}\varepsilon \\
(\%)\end{array}$ & $\begin{array}{c}\sigma \\
(\mathrm{MPa})\end{array}$ & $\begin{array}{c}\varepsilon \\
(\%)\end{array}$ & $\begin{array}{c}\sigma \\
(\mathrm{MPa})\end{array}$ & $\begin{array}{c}\varepsilon \\
(\%)\end{array}$ & $\begin{array}{c}\sigma \\
(\mathrm{MPa})\end{array}$ & $\begin{array}{c}\varepsilon \\
(\%)\end{array}$ \\
\hline $0^{*}$ & $\begin{array}{c}21,5 \\
\pm 1,26\end{array}$ & $\begin{array}{c}833 \\
\pm 82,8\end{array}$ & $\begin{array}{c}21,5 \\
\pm 1,26\end{array}$ & $\begin{array}{c}833 \\
\pm 82,8\end{array}$ & $\begin{array}{c}21,5 \\
\pm 1,26\end{array}$ & $\begin{array}{c}833 \\
\pm 82,8\end{array}$ & $\begin{array}{c}21,5 \\
\pm 1,26\end{array}$ & $\begin{array}{c}833 \\
\pm 82,8\end{array}$ & $\begin{array}{c}21,5 \\
\pm 1,26\end{array}$ & $\begin{array}{c}833 \\
\pm 82,8\end{array}$ \\
\hline 5 & $\begin{array}{l}18,3 \\
\pm 1,1\end{array}$ & $\begin{array}{c}700 \\
\pm 26,3\end{array}$ & $\begin{array}{l}20,4 \\
\pm 1,2\end{array}$ & $\begin{array}{c}748 \\
\pm 20,8\end{array}$ & $\begin{array}{l}19,9 \\
\pm 4,7\end{array}$ & $\begin{array}{c}710 \\
\pm 80,2\end{array}$ & $\begin{array}{l}20,7 \\
\pm 0,6\end{array}$ & $\begin{array}{c}713 \\
\pm 10,0\end{array}$ & $\begin{array}{l}17,7 \\
\pm 2,3\end{array}$ & $\begin{array}{c}642 \\
\pm 38,9\end{array}$ \\
\hline 10 & $\begin{array}{l}16,4 \\
\pm 1,2\end{array}$ & $\begin{array}{c}675 \\
\pm 29,9\end{array}$ & $\begin{array}{l}21,0 \\
\pm 0,6\end{array}$ & $\begin{array}{c}649 \\
\pm 15,6\end{array}$ & $\begin{array}{l}20,6 \\
\pm 0,9\end{array}$ & $\begin{array}{c}740 \\
\pm 13,7\end{array}$ & $\begin{array}{l}21,7 \\
\pm 1,1\end{array}$ & $\begin{array}{c}673 \\
\pm 65,1\end{array}$ & $\begin{array}{l}17,5 \\
\pm 4,4\end{array}$ & $\begin{array}{c}575 \\
\pm 86,4\end{array}$ \\
\hline 15 & $\begin{array}{c}20,2 \\
\pm 1,58\end{array}$ & $\begin{array}{c}600 \\
\pm 38,2\end{array}$ & $\begin{array}{l}19,1 \\
\pm 1,7\end{array}$ & $\begin{array}{c}538 \\
\pm 26,2\end{array}$ & $\begin{array}{l}22,3 \\
\pm 1,6\end{array}$ & $\begin{array}{c}674 \\
\pm 38,3\end{array}$ & $\begin{array}{l}23,7 \\
\pm 1,0\end{array}$ & $\begin{array}{c}645 \\
\pm 40,2\end{array}$ & $\begin{array}{l}22,1 \\
\pm 0,7\end{array}$ & $\begin{array}{c}660 \\
\pm 11,4\end{array}$ \\
\hline 20 & $\begin{array}{l}19,3 \\
\pm 1,0\end{array}$ & $\begin{array}{c}544 \\
\pm 25,2\end{array}$ & - & - & $\begin{array}{l}23,2 \\
\pm 1,3\end{array}$ & $\begin{array}{c}619 \\
\pm 42,6\end{array}$ & $\begin{array}{l}23,8 \\
\pm 0,4\end{array}$ & $\begin{array}{c}564 \\
\pm 25,2\end{array}$ & $\begin{array}{l}18,9 \\
\pm 2,7\end{array}$ & $\begin{array}{c}583 \\
\pm 81,9\end{array}$ \\
\hline 25 & $\begin{array}{l}18,3 \\
\pm 1,1\end{array}$ & $\begin{array}{c}488 \\
\pm 36,1\end{array}$ & $\begin{array}{r}19,5 \\
\pm 0,758\end{array}$ & $\begin{array}{c}446 \\
\pm 13,6\end{array}$ & $\begin{array}{l}22,7 \\
\pm 0,8\end{array}$ & $\begin{array}{c}537 \\
\pm 35,0\end{array}$ & - & - & - & - \\
\hline 30 & $\begin{array}{l}20,5 \\
\pm 0,8\end{array}$ & $\begin{array}{c}409 \\
\pm 11,1\end{array}$ & $\begin{array}{l}20,5 \\
\pm 1,4\end{array}$ & $\begin{array}{c}402 \\
\pm 18,7\end{array}$ & $\begin{array}{l}21,5 \\
\pm 1,6\end{array}$ & $\begin{array}{c}452 \\
\pm 19,1\end{array}$ & - & - & - & - \\
\hline
\end{tabular}

*Dados referentes à borracha natural sem compostos condutivos

preparadas por solução de SEBS com polianilina, onde Mantovani ${ }^{[31]}$ verificou que a incorporação de polianilina provoca alguma diminuição na resistência mecânica da blenda. Em um trabalho recente ${ }^{[32]}$, sobre as propriedades mecânicas dos compósitos de SEBS com compostos condutivos contendo polímero condutor (KP20 e KPY), verificou-se que a incorporação de 30phr desses compostos também causa diminuição da deformação e da tensão de ruptura em relação ao SEBS puro.

As curvas de Força $(\mathrm{N})$ versus deformação específica $(\varepsilon \%)$, representativas do comportamento dos compósitos de borracha natural com composição variando de $5,10,15,20,25$, e $30 \mathrm{phr}$ de negro de fumo ou composto condutivo, são mostradas nas Figuras 4, 5 e 6. Nota-se que o módulo aumenta com o aumento da quantidade de negro de fumo ou composto condutivo na matriz de NR e por conseguinte, ocorre uma diminuição na elongação percentual, com o aumento da quantidade de negro de fumo ou composto condutivo. Este mesmo comportamento foi observado para todos os compostos investigados neste trabalho. Estes resultados são consistentes com as curvas de torque, discutidas anteriormente, onde o aumento do conteúdo de composto condutivo promove um aumento no torque, devido a um aumento do reforçamento. Os resultados de tração demonstram que os negros de fumo e os com- postos condutivos atuam realmente como reforço para a matriz de NR, melhorando suas propriedades mecânicas como o módulo elástico e a tensão na ruptura sem no entanto perder significativamente sua capacidade de deformação. Segundo Sau et alli ${ }^{[33]}$, a mobilidade molecular do polímero diminui com o aumento do conteúdo do negro de fumo, devido à formação de ligações físicas entre as partículas das cargas condutivas e as cadeias do polímero. Consequentemente, a elongação na ruptura diminui com o aumento do conteúdo das cargas condutivas.

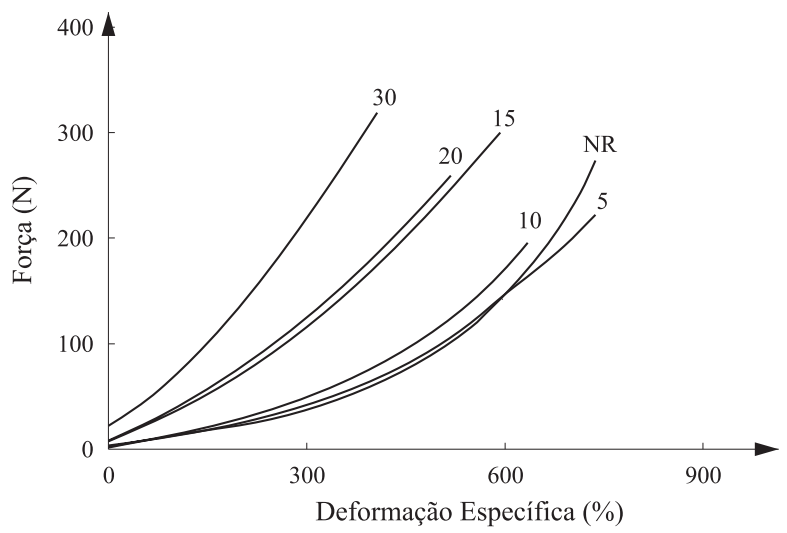

Figura 4. Curvas de força $(\mathrm{N})$ versus deformação específica (\%) para a borracha natural (NR) e para o compósito NR/KPY com 5, 10, 15, 20 e $30 \mathrm{phr}$ de composto condutivo. 


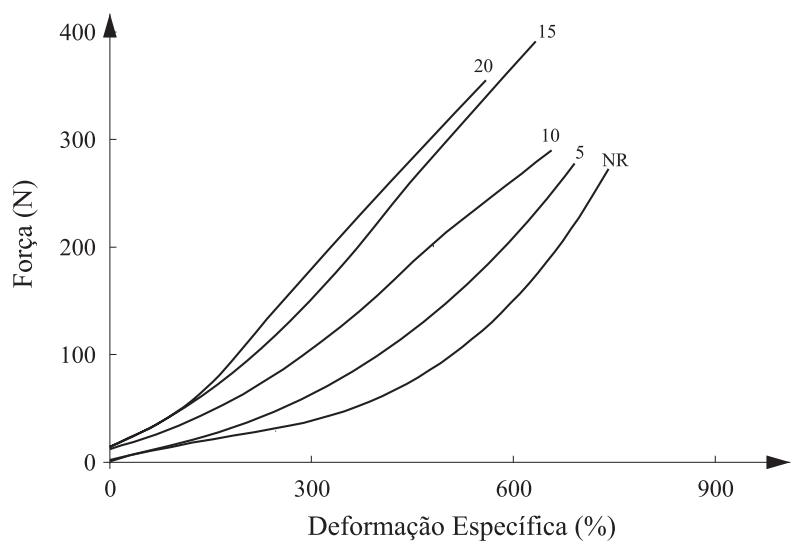

Figura 5 - Curvas de força $(\mathrm{N})$ versus deformação específica (\%) para a borracha natural (NR) e para o compósito NR/KP0 com 5, 10, 15 e 20phr de composto condutivo.

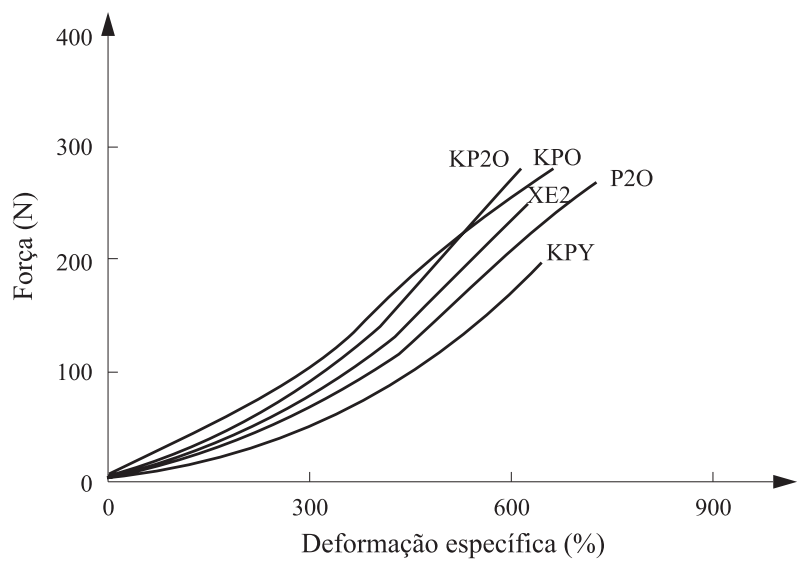

Figura 6- Curvas de força (N) versus deformação específica (\%) para a borracha natural (NR) e para os compósitos contendo 10phr dos diferentes tipos de compostos condutivos, conforme indicado.

A Figura 6 apresenta o comportamento das curvas de força $(\mathrm{N})$ versus deformação específica para a composição de 10phr dos diferentes compósitos NR/KPY, NR/KP20, NR/P20, NR/KP0 e NR/XE2. Verifica-se que as curvas apresentam formas semelhantes até a deformação de $\approx 400 \%$. O compósito NR/KP0 apresenta o maior módulo e a maior elongação, em relação aos outros compósitos. Estes resultados corroboram o que foi observado nas curvas de torque anteriormente, onde os compósitos com negro de fumo reforçam mais a NR do que os compósitos com polímero condutor, pelo fato de estes se localizarem na superfície do negro de fumo e reduzirem os "sítios" disponíveis para o ancoramento das moléculas da NR.

\section{Conclusões}

Os estudos realizados neste trabalho demonstraram a viabilidade da preparação de compósitos con- dutores elétricos de borracha natural com compostos condutivos contendo polímeros condutores via processamento a quente. Os compósitos com os compostos condutivos de negro de fumo contendo polímero condutor apresentaram valores de torque significativamente reduzidos em relação aos compósitos com negro de fumo. Este resultado representa uma vantagem dos compostos condutivos com polímero condutor, pois estes facilitam o processamento dos compósitos, melhorando os parâmetros de fluxo do fundido.

Dentre os compósitos estudados os compósitos de NR com negro de fumo KP0 foram os que apresentaram os maiores valores de condutividade elétrica e menor limite de percolação, em relação aos compósitos de NR com compostos contendo polímero condutor. Contudo, a condutividade elétrica dos compósitos de NR com os compostos com polímero condutor (NR/KP20 e NR/KPY) está na faixa dos materiais semicondutores elétricos com larga faixa de aplicação para esses compósitos. Estes resultados são interessantes e promissores se comparados com os dos compósitos condutores de negro de fumo com algumas borrachas e os de algumas blendas condutoras de polímeros convencionais com polianilina e polipirrol. As análises térmicas demonstraram que os compósitos com composto condutivo contendo polímero condutor são termicamente mais estáveis (até $\approx 330^{\circ} \mathrm{C}$ ) em relação aos compósitos com negro de fumo. Esta estabilidade térmica é superior à encontrada na literatura para os polímeros condutores puros e para as blendas e compósitos com polímeros condutores. O negro de fumo e os compostos contendo polímero condutor atuam também como reforço para a NR, melhorando suas propriedades mecânicas.

\section{Agradecimentos}

Os autores agradecem o apoio financeiro dado pela FAPESP, CNPq e CAPES.

\section{Referências Bibliográficas}

1. Stenger-Smith, J. D. - Prog. Polym. Sci., 23, p.57 (1998).

2. Zoppi, R.A. \& De Paoli, M. A. - Química Nova, 16, p.560 (1993).

3. Mattoso, L. H. C. - "Plásticos que Conduzem 
Eletricidade: Ficção ou Realidade?" Polímeros: Ciência e Tecnologia, p. 6-10, Jul/Set (1996).

4. Soares, B. G.; Ferreira, A. J. B. \& Camargo Jr. - S. Polímeros: Ciência e Tecnologia, Ano VIII, p.61 (1998).

5. Mattoso, L. H. C.; Malmonge, L. F.; Mantovani, G. L. \& MacDiarmid, A. G. - Current Trends in Polymer Science, 3, p.100 (1998).

6. Karásek, L.; Meissner, B.; Asai, S. \& Sumita, M. Polym. J. 28, p121 (1996).

7. Wessling, B. - Dispersion as the Key to Processing Conductive Polymer, In:Skothein, T. A.; Elsenbaumer, R. L. \& Reynolds, J. R. Handbook of Conducting Polymers. N.Y.: Marcel DecKer Inc, p.467-530. (1998)

8. Malmonge, L. F. \& Mattoso, L. H. C. - Polymer, 41, p.8387 (2000).

9. Shacklette, L. W.; Han, C. C. \& Luly, M. H. - Synth. Met., 55-57, p.3532 (1993).

10. Chiang, J. C. \& MacDiarmid, A. G. - Synth. Met., 13, p.193 (1986).

11. MacDiarmid, A. G. \& Epstein A. J. - Faraday Discuss., Chem. Soc. 88, p.317 (1989).

12. MacDiarmid, A. G.; Chiang, J. C.; Halpern, M.; Huang, W.S.; MU, S. L.; Somasiri, N. L. D.; Wu, W.; Yaniger, S.I. - Mol. Cryst. Liq. Cryst., 121, p. 173 (1985).

13. Avlyanov, J. K. \& Dahman, S. - Thermally stable, intrinsically conductive polymer-carbon black composites as new additives for plastics, American Chemical Society Meeting, 215:271-POLY, Part2,1998.

14. Avlyanov, J. K. - Synth. Met. 102, p.1272 (1999).

15. Zucolotto, V.; Avlyanov, J. K, Gregório, R. F. Mattoso, L. H. C. - Melt processing of conductive polymeric composites of PDVF and Eeonomers, Int. Conf. Sci. Tech. Synthetic Metals, p.171.Gastein/Austria, Jul (2000).

16 Karásek, L. \& Sumita, M. J. - Mat. Sci., 31, p. 281 (1996).

17. Aik-Hwee \& Tanaka, Y. - Polym. Sci., 3, p.493 (1993).

18. Mwila, J.; Miraftab, M. \& Horrocks, A. R. - Polym. Degrad. and Stab., 44, p. 351 (1994).
19 Tang, H.; Chen, X.; Tang, A. \& Luo, Y. J. - Appl. Polym. Sci., 59, p. 383 (1996).

20. Ahmad, S.; Abdullah, I.; Sulaiman, C. S.; Kohijiya, S. \& Yoon, J. R., - J. Appl. Polym. Sci., 51, p. 1357 (1994).

21. Tang, H.; Chen,X. \& Luo, Y. - Eur. Polym. J., 32, p. 963 (1996).

22. Ikkala, O. T.; Laakso, J.; Vakiparta, K.; Virtanen, E.; Ruohonen, H.; Jarvinen, H.; Taka, T.; Passiniemi, P.; Osterholm, J. E.; Cao, Y.; Andreatta, A.; Smith, P. \& Heeger, A. J. - Synth. Met., 69, p. 97 (1995).

23. Ikkala, O. T.; Lindholm, T. M.; Ruohonen, H.; Selantaus, M. \& Vakiparta, K. - Synth. Met., 69, p.135 (1995).

24. Omastová, M.; Pionteck, J. \& Kosina, S. - Eur. Polym. J., 32, p.681 (1996).

25. Jesus, M. C. \& Weiss R. A. - Macromolecules, 31, p.2230 (1998).

26. Faez, R.; Rezende, M. C.; Martin, I. M.; \& De Paoli, M. A. - Polímeros: Ciência e Tecnologia, 10, p.130 (2000).

27. Iwamoto, N. - Composite adhesive enhancements for the microelectronics packaging industry using conductive polymers, in: $6^{\text {th }}$ Annual Int. conf. Composites Eng. Orlando/Florida, Jul (1999)

28 Mattoso, L. H. C. - Química Nova, 19, p.388 (1996)

29. Wei, Y. \& Hsueh, K. F., J. - Appl. Polym. Sci. Part A: Polym. Chem., 27, p. 4351 (1989).

30. Vikki, T.; Pietila, L. O.; Osterholm, H.; Ahjopalo, L.; Takala, A.; Toivo, A.; Levon, K.; Passiniemi P. \& Ikkala, O. - Macromolecules, 29, p.2945 (1996).

31. Mantovani, G.L., Mattoso, L.H.C., \& MacDiarmid, A.G. Synth. Met., 84, p.73 (1997).

32. Zucolotto, V. - Processamento por fusão de compósitos poliméricos condutores elétricos, Dissertação de mestrado, Universidade Federal de São Carlos, PPGCEM, Brasil (1999).

33. Sau, K. P.; Chaki, T. K. \& Khastgir, D. J. - Mat. Sci., 32, p. 5717 (1997).

Recebido: 13/06/01

Aprovado: 01/10/01 